\title{
Expressão de MMPs, marcadores angiogênicos e proliferação celular em tumores odontogênicos
}

Primeira submissão em 28/03/12 Última submissão em 12/07/12 Aceito para publicação em $27 / 07 / 12$ Publicado em 20/10/12

\section{Expression of MMPs, angiogenic and proliferation cell markers in odontogenic tumors}

Felipe Rodrigues de Matos'; Rodrigo Gadelha Vasconcelos²; Lélia Maria Guedes Queiroz ; Roseana de Almeida Freitas ${ }^{3}$

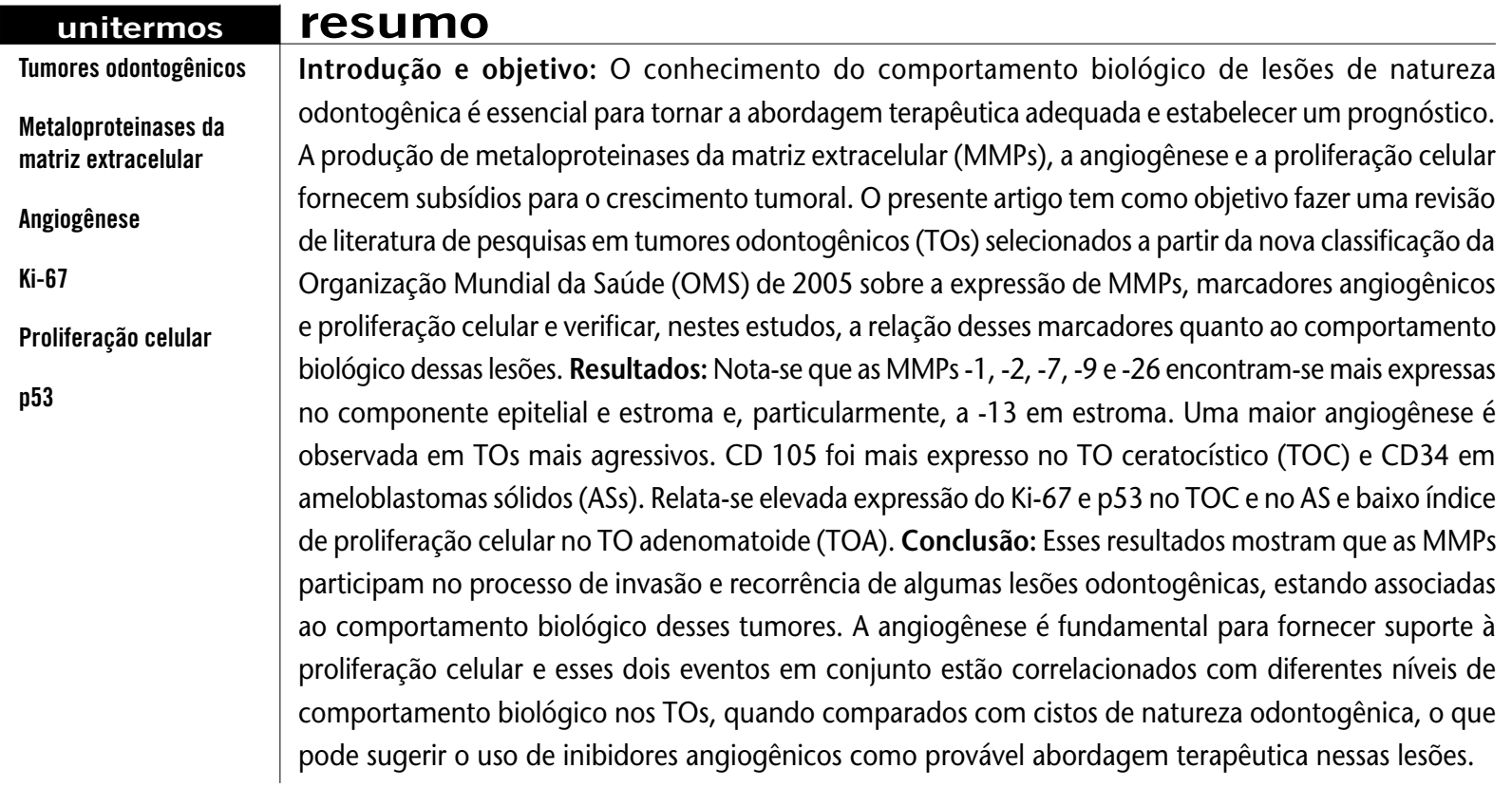

abstract

Introduction and objective: The study of biological behavior of odontogenic lesions is essential to the establishment of appropriate therapeutic approach and prognosis. The production of extracellular matrix metalloproteinases (MMPs), angiogenesis and cell proliferation contribute to tumor growth. This paper aims to review the literature on odontogenic tumors (OT) selected according to the new World Health Organization classification (WHO- 2005) by evaluating the expression of MMPs, angiogenic and cell proliferation. Furthermore, it aims to verify the relation between these markers and the biological behavior of these lesions. Results: it was found that MMPs $-1,-2,-7,-9$ and -26 had a higher expression in both epithelial component and stroma, and 13 particularly in the stroma. Increased angiogenesis was observed in more aggressive OT. CD105 expression was higher in keratocystic odontogenic tumour (KOT) and CD34 in solid ameloblastomas (SA). It was observed a higher expression of Ki-67 and p53 in SA and KOT and a low cell proliferation rate in the adenomatoid odontogenic tumour (AOT). Conclusion: These results show that MMPs are involved in invasion and recurrence of some odontogenic lesions and are associated with the biological behavior of these tumors. Angiogenesis is critical to provide support to cell proliferation and these concomitant events are correlated with different levels of biological behavior in OT when compared to odontogenic cysts, hence the use of angiogenic inhibitors may be a potential therapeutic approach in these lesions. key words

Odontogenic tumour

Matrix metalloproteinases

Angiogenesis

Ki-67

Cell proliferation

p53

1. Cirurgião-dentista; mestre em Patologia Oral; doutorando do Programa de Pós-graduação em Patologia Oral da Universidade Federal do Rio Grande do Norte (UFRN).

2. Cirurgião-dentista; mestre em Odontologia; doutorando do Programa de Pós-graduação em Patologia Oral da UFRN.

3. Doutora em Patologia Oral; professora do Programa de Pós-graduação em Patologia Oral da UFRN. 


\section{Introdução}

Os tumores odontogênicos (TOs) são lesões derivadas dos elementos epiteliais, mesenquimais ou ectomesenquimais, que ainda são ou tenham sido relacionados com odontogênese. Essas lesões podem ser de localização intra ou extraóssea. Algumas representam neoplasias verdadeiras, podendo, raramente, exibir comportamento maligno, enquanto outras representam malformações semelhantes a tumor (hamartomas). O odontoma tem sido relatado como o mais comum de todos os TO, seguido do ameloblastoma, que é o segundo mais comum ${ }^{(3)}$.

O consenso prévio sobre a taxonomia dos TOs, cistos e lesões associadas confirmou que as relações morfológicas e as indutoras características entre as várias partes do germe dentário normal são reproduzidas, em maior ou menor expressão, em muitos dos tumores e lesões similares a tumores dos tecidos odontogênicos ${ }^{(3)}$.

O conhecimento do comportamento biológico de entidades patológicas que afetam a cavidade oral, incluindo os TOs, é essencial para a seleção de uma abordagem terapêutica mais adequada e o estabelecimento de um prognóstico mais preciso. Tal fato vem despertando o interesse de pesquisadores em investigar, por meio de variedades metodológicas diferentes, os aspectos relacionados com a biologia molecular das populações de células tumorais em uma tentativa de elucidar pontos que ainda permanecem obscuros ${ }^{(2)}$.

Nesse contexto, as metaloproteinases de matriz extracelular (MMPs) são enzimas que desempenham papel importante na regulação da integridade, composição e degradação da matriz extracelular (MEC), tendo sido relacionadas, dessa forma, com a tumorigênese ${ }^{(35)}$.

Adicionalmente, os tecidos neoplásicos necessitam de oxigênio e nutrientes para a sua sobrevivência e crescimento celular, induzindo, mediante essa necessidade, a formação de novos vasos sanguíneos por meio da angiogênese. Fatores relacionados com indução de angiogênese têm sido utilizados para definir a agressividade e a invasão tumoral. Assim, uma densa rede vascular assegura o processo metabólico necessário e eficaz para as células tumorais ${ }^{(11)}$. A atividade proliferativa dessas células também tem sido relatada como importante marcador prognóstico e indicador de agressividade biológica. A atividade proliferativa de uma neoplasia vem sendo normalmente avaliada por meio da mensuração da proporção de células comprometidas com o ciclo celular, de modo a avaliar o potencial de crescimento intrínseco da lesão(12).
Em TOs, têm-se investigado a imunolocalização de proteínas do ciclo celular associadas a essas lesões. Proliferação, organização, diferenciação celular, angiogênese e reação indutiva de um tecido sobre o outro são parâmetros importantes que devem ser levados em consideração durante o desenvolvimento dessas neoplasias ${ }^{(26)}$.

Diante do exposto, o presente estudo tem como objetivo fazer uma revisão de literatura de pesquisas em TO selecionados a partir da nova classificação da Organização Mundial da Saúde (OMS) de 2005 sobre a expressão de MMPs, marcadores angiogênicos e proliferação celular e verificar, nesses estudos, a relação desses marcadores quanto ao comportamento biológico dessas lesões.

\section{Revisão de literatura}

\section{MMPs em Tos}

Recentemente, tem sido proposto que as MMPs exercem importante papel na regulação da integridade $\mathrm{e}$ composição da MEC em cistos e do TO ${ }^{(17)}$. As MMPs são uma família de enzimas proteolíticas zinco-dependentes, capazes de degradar todos os componentes da MEC. Até 0 momento, na família de MMPs, estão incluídos pelo menos 24 membros bem caracterizados, os quais são classificados em colagenases, gelatinases, matrilisinas, estromelisinas e MMP tipo membrana. A clivagem da membrana basal e da MEC pelas proteinases das células tumorais é pré-requisito para invasão tumoral ${ }^{(34)}$.

Estudos imuno-histoquímicos reportaram a expressão de MMP-1, -2, -7, -9 e -26, além dos inibidores teciduais de metaloproteinase (TIMP)-1 e -2 em ameloblas-

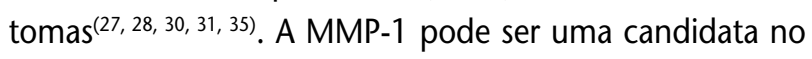
processo da progressão tumoral, visto que foi encontrada maior expressão da dela no ameloblastoma sólido (AS), quando comparada com o TO ceratocístico (TOC). Tal achado poderia ser explicado pela presença da tenascina, principal substrato da MMP-1, na porção central das ilhas tumorais que lembram o retículo estrelado do órgão do esmalte ${ }^{(28)}$. Não obstante, outro estudo constatou maior expressão de MMP-2 em AS em relação ao TOC. Esse resultado sugere que o aumento da expressão da MMP-2 no AS poderia estar relacionado com um comportamento mais infiltrativo e agressivo.

As matrilisinas (MMP-7 e -26) estão envolvidas na proliferação celular, na apoptose, na invasão e na metástase. Elas diferem das outras MMPs pelo baixo peso molecular e pela 
falta do domínio C-terminal hemopexina ${ }^{(32)}$. Ambas foram expressas em AS, tanto no componente epitelial quanto no estromal. De maneira particular, a expressão da MMP-26 foi homogeneamente distribuída nos ameloblastomas plexiformes, enquanto no padrão folicular foi observada na porção central dos ninhos epiteliais. Nos TOs adenomatoides (TOA), ambas as matrilisinas foram evidentes, tanto nas células colunares e cuboidais das estruturas ductiformes como nos ninhos tumorais, nos fibroblastos e nas células endoteliais no estroma. Apesar de não ter sido constatada diferença na imunoexpressão das referidas proteínas entre essas lesões, Souza Freitas et al. ${ }^{(32)}$ sugerem a participação da MMP-7 e - 26 no processo de invasão dos ameloblastomas, pois a forte expressão verificada nelas pode estar associada à ativação de MMP-2 e -9.

Adicionalmente, a MMP-9 e o TIMP-2 podem cooperar com a progressão tumoral do AS, refletindo no seu comportamento clínico. Siqueira et al.(31) observaram que os AS exibiram expressão imuno-histoquímica da MMP-9 e do TIMP-2 maior quando comparados com o TO cístico calcificante (TOCC), que é uma lesão não invasiva com componente epitelial ameloblastomatoso. Outra função biológica discutida da MMP-9 é a sua participação no processo de reabsorção óssea, atuando na degradação de componentes orgânicos da matriz óssea não mineralizada, como colágeno tipo I e II(21). Em sistema de cocultura de ameloblastoma, verificou-se expressão de MMP-9, mas, com a adição do TIMP-1 ao sistema, não houve redução significativa na reabsorção óssea. Assim, a MMP-9 poderia participar, entretanto não seria a principal protease envolvida na degradação da matriz óssea ${ }^{(27)}$.

No campo da biologia molecular, identificaram-se maiores níveis de ácido ribonucleico mensageiro (RNAm) da MMP-2 em AS comparados com os do TOC ${ }^{(35)}$, bem como da MMP-14 e do TIMP-2 em ameloblastomas recorrentes com ameblastomas primários e AS comparados com ameloblastomas unicísticos $(\mathrm{AU})^{(34)}$. Esses achados reforçam o comportamento agressivo do ameloblastoma, sugerindo que as MMPs podem participar na invasão e na recorrência desse tumor, além de mediarem a reabsorção óssea. Os TIMPs, embora tenham habilidade de inibir a atividade das MMPs, poderiam desempenhar efeitos biológicos adicionais, como regulação da proliferação celular, migração e apoptose.

Entretanto, em um modelo experimental animal(36) foram observados achados díspares. Foi realizado xenotransplante de ameloblastoma com transfecção do gene da TIMP-2. Posteriormente, mensurou-se o RNAm e a expressão proteica da MMP-2 e do TIMP-2. Os pesquisadores verificaram que no grupo de animais transfectados houve redução no crescimento tumoral, aumento dos níveis de RNAm e proteico do TIMP-2 e redução da expressão proteica da MMP-2. Esta é ativada por meio da formação de um complexo ternário intermediário da MMP-14, TIMP-2 e pró-MMP-2. O TIMP-2 expresso nas áreas de interação entre células epiteliais e mesenquimais poderia ser regulador do crescimento celular e da invasão tumoral.

Em TOA, lesão menos agressiva e com potencial proliferativo menor quando comparado com ameloblastoma, tem sido reportada expressão imuno-histoquímica da MMP-1, -2 e -9 em células do parênquima e estroma da lesão, destacando-se a forte expressão da MMP-1 em células tumorais estromais. A menor expressão da MMP-2 poderia estar relacionada com a necessidade de preservar pelo menos alguns dos constituintes da membrana basal, crucial para o processo de diferenciação das células neoplásicas, bem como para a manutenção da arquitetura estrutural nos ninhos tumorais. A MMP-9 finalizaria a degradação do colágeno iniciada pela MMP-1 ${ }^{(28)}$.

O TOC possui comportamento biológico agressivo, com marcada tendência a recorrência ${ }^{(6,18)}$. Verifica-se nessa lesão elevada atividade mitótica epitelial na camada suprabasal. As células estromais são as principais fontes de MMPs e as MMP-1 e - 13 poderiam se destacar nesse contexto. Leonardi et al. ${ }^{(18)}$ analisaram a expressão de MMP-13 em TOC sindrômicos e não sindrômicos e constataram que nos primeiros há proeminente expressão da MMP-13 em estroma quando comparada com componente epitelial, enquanto nos TOC não sindrômicos a expressão da MMP-13 foi ausente na maioria dos casos. Cavalcanti et al. ${ }^{(6)}$ verificaram maior expressão de MMP-1 no componente estromal de TOC sindrômicos quando comparados com os não sindrômicos. A superexpressão de MMPs no estroma dos TOC poderia elucidar a agressividade dessas lesões.

O estroma representa fonte de MMPs e a expressão dessas enzimas é super-regulada sob determinadas condições que poderiam promover o crescimento da lesão por meio da degradação de tecidos adjacentes. Uma possível explicação para uma maior expressão de MMPs em TOC sindrômicos seria a hiperativação da via de sinalização WNT/SHH. Mutações no gene human patched (PTCH) levariam à maior expressão de $\beta$-catenina que, por sua vez, se ligaria a fatores de transcrição lymphoid enhancer factor/T-cell factor (LEF/TCF), ativando genes das $\mathrm{MMPs}^{(8)}$, o que sugere que as MMPs liberadas pelas células estromais poderiam potencializar a ação das MMPs produzidas pelo componente epitelial(32). 
Nos mixomas odontogênicos, foi verificada expressão imuno-histoquímica da MMP-1 na MEC em 66,6\% dos casos, semelhantemente ao observado para a MMP-2 $(58,3 \%)$. Cerca de $33 \%$ dos casos foram imunopositivos à MMP-9. A MMP-1 seria a principal enzima envolvida na degradação da MEC, juntamente com a MMP-2 em menor proporção, agindo em combinação com a MMP-9 nos fragmentos desnaturados derivados da degradação do colágeno tipo I. Essas proteases poderiam participar da invasão local dos mixomas odontogênicos por meio de um coordenado mecanismo entre elas na degradação eficiente da $\mathrm{MEC}^{(25)}$.

De forma geral, observa-se na literatura que o estroma desempenha papel importante no microambiente tumoral, o que favorece a progressão da lesão, evidenciada pela sinergismo da cooperação entre as células epiteliais e os fibroblastos estromais na produção de $\mathrm{MMPs}^{(32)}$. De acordo com Lynch e Matrisian ${ }^{(19)}$, as MMPs produzidas pelas células tumorais e estromais podem processar moléculas da superfície celular, proteínas, fatores de crescimento e citocinas presentes na MEC, provocando alterações no microambiente tumoral, as quais favorecem o crescimento tumoral, a migração, a invasão e a angiogênese.

\section{Angiogênese}

Tecidos neoplásicos necessitam de oxigênio e nutrientes para sua sobrevivência e seu crescimento e isso propicia a formação de novos vasos por meio da angiogênese para suprir a demanda e remover a grande quantidade de produtos metabólicos $^{(7)}$. A angiogênese tem sido avaliada em várias lesões humanas, incluindo câncer de mama, carcinoma de células escamosas de cabeça e pescoço e TO ${ }^{(11)}$.

O CD105 é uma glicoproteína associada à formação de novos vasos. Utilizando esse marcador por meio da imuno-histoquímica, verificou-se uma maior densidade microvascular nos TOC, bem como vasos sanguíneos com morfologia levemente aberrante e lúmens tortuosos e dilatados, quando comparados com cisto dentígero e mucosa oral normal. Esses achados sugerem que a angiogênese pode estar relacionada com o aumento do metabolismo tecidual e a necessidade nutricional do epitélio proliferativo ${ }^{(11)}$. Tal assertiva é suportada por achados ultraestruturais dos vasos sanguíneos do TOC, nos quais se verificou presença de trombose mais frequente quando comparada com cisto dentígero. A presença de trombose nos vasos, que conteria grande número de plaquetas, poderia produzir fatores angiogênicos, como fator de crescimento endotelial vascular (VEGF), fator de crescimento fibroblástico (FGF) e fator de crescimento transformante-beta (TGF- $\beta$ ), que induziriam à proliferação e à migração de células endoteliais para formar os novos leitos capilares ${ }^{(10)}$. Adicionalmente, a maior presença de miofibroblastos nessa lesão constituiria uma fonte extra na produção de proteínas angiogênicas, bem como proteinases de degradação da MEC, que juntas favoreceriam a invasão e o crescimento das células neoplásicas do TOC ${ }^{(33)}$.

A glicoproteína CD34 é outra molécula associada ao tecido vascular presente em vasos sanguíneos imaturos e tem sido utilizada para mensurar a angiogênese, embora não faça distinção entre vasos sanguíneos neoformados e quiescentes ${ }^{(29)}$. Um estudo em ameloblastomas revelou a maior média de densidade microvascular (MVD) em ameloblastomas sólidos, seguida do TO epitelial calcificante (CEOT), ameloblastoma unicístico e cisto dentígero. Em ameloblastomas unicísticos, a média do número de vasos imunorreativos ao CD34 foi maior no tecido estromal em torno das ilhas ameloblásticas intramurais, quando comparada com as adjacentes ao epitélio cístico(1).

Achados semelhantes foram observados por Chen et al.(7) e Seifi et al.(29), os quais verificaram maior MVD em ameloblastoma sólido, seguido pelo TOC. Constatou-se maior MVD na região intratumoral dos ameloblastomas que na área peritumoral, provavelmente decorrente do efeito indutor do epitélio odontogênico tumoral.

Os vasos sanguíneos no estroma são um dos fatores essenciais para o crescimento epitelial ${ }^{(29)}$. O estroma do ameloblastoma é uma rica fonte de fator de crescimento da célula endotelial derivado de plaqueta e angiopoetina-1 e -2 , sendo as angiopoetinas também produzidas pelas células epiteliais tumorais. Dessa forma, sugere-se que essas moléculas induzem a angiogênese, destacando-se a angiopoetina-1 que atua sinergicamente com o $\operatorname{VEGF}{ }^{(16)}$. Paralelamente, a produção de óxido nítrico induzida pelo óxido nítrico sintase (iNOS) aumenta a expressão e a síntese de VEGF, sobretudo pelo aumento da atividade do promotor para o gene do VEGF. Os resultados de Chen et al.(7) reforçam essa assertiva, uma vez que verificaram maior expressão de VEGF e iNOS em ameloblastomas sólidos do que em CEOT, aumentando o suprimento de vasos sanguíneos necessários para a manutenção da proliferação epitelial.

\section{Proliferação celular}

Um marcador mais específico para células em proliferação é o Ki-67, um antígeno que é rapidamente degradado após a mitose. Esse marcador é uma proteína não histônica, inicialmente expressa em Gap 1 (G1), que apresenta níveis 
aumentados em síntese (S) e Gap 2 (G2) e pico em mitose $(\mathrm{M})$, mas ausente nas células em repouso (G0). Tem-se discutido que a marcação para o Ki-67 é mais precisa do que a contagem do número de figuras de mitose ou proliferating cell nuclear antigen (PCNA) positivo(13).

Gong et al. ${ }^{(13)}$ investigaram as características histopatológicas e a expressão do Ki-67 em TOCC, tumores dentinogênicos de células fantasmas (TDCF) e carcinomas odontogênicos de células fantasmas (COCF). Nestes, a expressão de Ki-67 foi significativamente maior do que em TOCC, TDCF e ameloblastoma, reforçando a natureza maligna do COCF. Além disso, houve uma tendência de menor expressão de Ki-67 no TOCC, comparado com o TDCF, e menor expressão quando comparado com ameloblastoma. Esses resultados tomados juntos sugerem similaridade quanto ao comportamento biológico do TDCF, mais com ameloblastoma do que com TOCC. Neville et al. ${ }^{(24)}$ abordam, em seu livro, TOCC como cisto odontogênico, embora ele esteja incluído na última classificação da OMS de $2005^{(3)}$ como TO. Dessa forma, os resultados do estudo supracitado ${ }^{(13)}$ poderiam, em parte, fundamentar uma reflexão para um possível retorno dessa entidade no grupo dos cistos odontogênicos.

Um grupo de pesquisadores verificou a imunoexpressão de Ki-67 nas quatro variantes clínicas de amelobastoma e em carcinoma ameloblástico (CA $)^{(4,5)}$. O índice de expressão do Ki-67 no CA foi de 41,2\%; no AU 15,1\%; no AS 12,8\%; no ameloblastoma periférico (AP) 6,4\%; e no desmoplásico (AD) 1,5\%. Os resultados sugerem que o aumento do índice de Ki-67 observado em CA está de acordo com sua maior agressividade, quando comparado com os ameloblastomas. Adicionalmente, foi observada uma tendência de menor índice de proliferação no AS em comparação com AU que, segundo os autores, possui menos células semelhantes ao retículo estrelado que o AS e, consequentemente, a maioria das células contadas correspondiam às células da camada basal e suprabasal que tendem ser mais positivas. Em outras palavras, a proporção desses diversos tipos de células epiteliais, bem como os diferentes mecanismos de crescimento do AU e do AS podem influenciar nos resultados do índice de proliferação. Curiosamente, o $\mathrm{AD}$ apresentou o menor índice de proliferação. Os autores sugerem que o AD difere dos outros tipos de ameloblastomas intraósseos e a realização de mais estudos para compreender o papel do estroma desmoplásico no comportamento dessa neoplasia se faz necessária.

O gene p53, conhecido como "guardião do genoma", é mais frequentemente mutado em cânceres humanos, incluindo o câncer oral. Para evitar a replicação de células com ácido desoxirribonucleico (DNA) danificado, a proteína p53 provoca uma parada do ciclo celular para reparo do DNA ou apoptose no limite G1-S do ciclo celular. Consequentemente, a perda da função do p53 em células o torna suscetível a acumular uma série de defeitos genéticos em uma taxa aumentada, resultando em progressão e transformação maligna. Em células de tumores malignos, anormalidades do gene p53 podem levar à perda transcricional do produto p53 ou à superexpressão da proteína p53 mutante, funcionalmente inativa. A perda da atividade transcricional do gene p53 é um dos primeiros eventos naturais do início do câncer, o que confere às células do tumor dois fenótipos mais importantes: 1) vantagem de crescimento para a expansão clonal e escape do ponto de checagem em G1 e apoptose; 2 ) instabilidade genômica e aquisição de mais mutações oncogênicas ${ }^{(9)}$.

Barboza et al. ${ }^{(2)}$ investigaram a expressão de proteínas PCNA e p53 em ameloblastomas e o TOA em relação ao importante papel desempenhado na proliferação célular e tumorigênese. Os resultados desse estudo sugerem que os padrões histológicos dos ameloblastomas não apresentaram correlação direta com o comportamento clínico e, consequentemente, com o prognóstico dos casos. Os resultados também indicam que o ameloblastoma apresenta maior potencial proliferativo que o TOA, o que pode contribuir para explicar suas características de maior agressividade e potencial invasivo.

A elevada expressão do Ki-67 tem sido observada no epitélio do TOC quando comparada com cistos de desenvolvimento e inflamatórios, mais particularmente na camada suprabasal(22). Gurgel et al.(14) analisaram a expressão imuno-histoquímica do Ki-67 e p53 em TOC. Os resultados demonstraram que o Ki-67 foi localizado principalmente nas camadas celulares suprabasais. Não houve diferença estatisticamente significante na imunoexpressão dessas proteínas entre a forma primária e a recorrente, ou entre TOC associados à síndrome do carcinoma nevoide basocelular e TOC esporádicos. Os autores concluíram que é possível que o comportamento biológico do TOC pode estar relacionado com a região suprabasal proliferativa no epitélio cístico, observado pelos altos níveis de Ki-67 e p53. Por outro lado, Gadbail et al. (12) verificaram uma maior positividade do Ki-67 e do p53 em ameloblastomas do que em TOC. Esses resultados sugerem que o aumento da expressão do p53 não necessariamente implicaria associação apenas com lesões odontogênicas malignas ${ }^{(23)}$, mas uma tendência de ser expressa em lesões císticas e TOs mais agressivos, podendo promover a proliferação celular nessas lesões ${ }^{(12)}$. 
lezzi et al.(15) avaliaram a expressão da proteína p53 e Ki-67 no mixoma odontogênico dos maxilares. Os resultados demonstraram que as células do epitélio odontogênico exibiram positividade de $5 \%$ a $10 \%$ para a p 53 e $1 \%$ para $\mathrm{Ki}-67$, enquanto as células estromais foram negativas para o p53 e 4\% positivas para o Ki-67. À semelhança, Martinez-Mata et al. ${ }^{(20)}$ encontraram índice de proliferação de $1 \%$. Essa maior atividade proliferativa estromal levaria à maior síntese e secreção de MEC, que bioquimicamente é rica em um componente com grande capacidade de se ligar à água: o glicosaminoglicano. Dessa forma, a natureza bioquímica da MEC seria responsável pelo crescimento tumoral do mixoma.

\section{Considerações finais}

Esses resultados mostram que as MMPs participam no processo de invasão e recorrência de algumas lesões odontogênicas, estando associadas ao comportamento biológico desses tumores. Paralelamente, os papéis exercidos pelos TIMPs nessas lesões ainda não estão bem esclarecidos em função de resultados conflitantes. A angiogênese é fundamental para fornecer suporte à proliferação celular e esses dois eventos em conjunto estão correlacionados com diferentes níveis de comportamento biológico nos TO, quando comparados com cistos de natureza odontogênica, o que pode sugerir o uso de inibidores angiogênicos como provável abordagem terapêutica nessas lesões.

\section{Referências}

1. ALAEDDINI, M. et al. Comparison of angiogenesis in keratocystic odontogenic tumours, dentigerous cysts and ameloblastomas. Oral Dis, v. 15, n. 6, p. 422-7, 2009.

2. BARBOZA, C. A. et al. Proliferating cell nuclear antigen (PCNA) and p53 protein expression in ameloblastoma and adenomatoid odontogenic tumor. Braz Dent J, v. 16, n. 1, p. 56-61, 2005.

3. BARNES, L. et al. (Eds.). World Health Organization Classification of Tumours: pathology and genetics of head and neck tumours. Lyon: IARC Press, v. 9, 2005. p.118-21

4. BOLOGNA-MOLINA, R. et al. Comparative expression of syndecan-1 and Ki-67 in peripheral and desmoplastic ameloblastomas and ameloblastic carcinoma. Pathol Int, v. 59, n. 4, p. 229-33, 2009.

5. BOLOGNA-MOLINA, R. et al. Syndecan-1 (CD138) and Ki67 expression in different subtypes of ameloblastomas. Oral Oncol, v. 44, n. 8, p. 805-11, 2008.

6. CAVALCANTE, R. B. et al. Immunohistochemical expression of MMPs 1, 7, and 26 in syndrome and nonsyndrome odontogenic keratocysts. Oral Surg Oral Med Oral Pathol Oral Radiol Endod, v. 106, n. 1, p. 99-105, 2008.

7. CHEN, W. L. et al. Expression of inducible nitric oxide synthase and vascular endothelial growth factor in ameloblastoma. The Journal of craniofacial surgery, v. 20, n. 1, p. 171-5; discussion 176-7, 2009.

8. DAKUBO, G. D.; MAZEROLLE, C. J.; WALLACE, V. A. Expression of Notch and Wnt pathway components and activation of Notch signaling in medulloblastomas from heterozygous patched mice. J Neurooncol, v. 79, n. 3, p. 221-7, 2006.

9. DEMIAN, N. et al. Malignant transformation of calcifying epithelial odontogenic tumor is associated with the loss of p53 transcriptional activity: a case report with review of the literature. J Oral Maxillofac Surg, v. 68, n. 8, p. 1964-73, 2010.

10. EL-LABBAN, N. G.; AGHABEIGI, B. A comparative stereologic and ultrastructural study of blood vessels in odontogenic keratocysts and dentigerous cysts. J Oral Pathol Med, v. 19, n. 10, p. 442-6, 1990.

11. GADBAIL, A. R. et al. Tumor angiogenesis in keratocystic odontogenic tumor assessed by using CD-105 antigen. J Oral Pathol Med, v. 40, n. 3, p. 263-9, 2011.

12. GADBAIL, A. R.; PATIL, R.; CHAUDHARY, M. Coexpression of $\mathrm{Ki}-67$ and p53 protein in ameloblastoma and keratocystic odontogenic tumor. Acta Odontol Scand, 2011.

13. GONG, Y. et al. The expression of NF-kappaB, Ki-67 and MMP-9 in CCOT, DGCT and GCOC. Oral Oncol, v. 45, n. 6, p. 515-20, 2009.

14. GURGEL, C. A. et al. Expression of Ki-67, p53 and p63 proteins in keratocyst odontogenic tumours: an immunohistochemical study. J Mol Histol, v. 39, n. 3, p. 311-6, 2008.

15. IEZZI, G. et al. MIB-1, Bcl-2 and p53 in odontogenic myxomas of the jaws. Acta Otorhinolaryngol Ital, v. 27, n. 5, p. 237-42, 2007.

16. KUMAMOTO, H.; OOYA, K. Immunohistochemical detection of platelet-derived endothelial cell growth factor/thymidine phosphorylase and angiopoietins in ameloblastic tumors. J Oral Pathol Med, v. 35, n. 10, p. 606-12, 2006.

17. LEONARDI, R.; CALTABiAnO, R.; LORETO, C. Collagenase-3 (MMP-13) is expressed in periapical lesions: an immunohistochemical study. Int Endod J, v. 38, n. 5, p. 297-301, 2005.

18. LEONARDI, R. et al. MMP-13 expression in keratocyst odontogenic tumour associated with NBCCS and sporadic keratocysts. Oral Dis, v. 16, n. 8, p. 795-800, 2010. 
19. LYNCH, C. C.; MATRISIAN, L. M. Matrix metalloproteinases in tumor-host cell communication. Differentiation, v. 70, n. 9-10, p. 561-73, 2002.

20. MARTINEZ-MATA, G. et al. Odontogenic myxoma: clinicopathological, immunohistochemical and ultrastructural findings of a multicentric series. Oral Oncol, v. 44, n. 6, p. 601-7, 2008.

21. MATOS, F. R. et al. Immunoexpression of MMP-9, VEGF, and $\mathrm{VWF}$ in central and peripheral giant cell lesions of the jaws. J Oral Pathol Med, v. 40, n. 4, p. 338-44, 2011.

22. MENDES, R. A.; CARVALHO, J. F.; VAN DER WAAL, I. A comparative immunohistochemical analysis of COX-2, p53, and Ki-67 expression in keratocystic odontogenic tumors. Oral Surg Oral Med Oral Pathol Oral Radiol Endod, v. 111, n. 3, p. 333-9, 2011.

23. MOTOSUGI, U. et al. Ghost cell odontogenic carcinoma arising in calcifying odontogenic cyst. Ann Diagn Pathol, v. 13, n. 6, p. 394-7, 2009.

24. NEVILLE, B. W. et al. Patologia oral e maxilofacial. Rio de Janeiro: Guanabara Koogan, 2009.

25. NONAKA, C. F. et al. Immunohistochemical expression of matrix metalloproteinases 1,2 , and 9 in odontogenic myxoma and dental germ papilla. Pathol Res Pract, v. 205, n. 7, p. 458-65, 2009.

26. PONTES, H. A. et al. Immunoexpression of Ki67, proliferative cell nuclear antigen, and $\mathrm{Bcl}-2$ proteins in a case of ameloblastic fibrosarcoma. Ann Diagn Pathol, v. 14, n. 6, p. 447-52, 2010.

27. QIAN, Y.; HUANG, H. Z. The role of RANKL and MMP-9 in the bone resorption caused by ameloblastoma. J Oral Pathol Med, v. 39, n. 8, p. 592-8, 2010.
28. RIBEIRO, B. F. et al. Immunoexpression of MMPs-1, -2, and -9 in ameloblastoma and odontogenic adenomatoid tumor. Oral Dis, v. 15, n. 7, p. 472-7, 2009.

29. SEIFI, S.; SHAFAIE, S.; GHADIRI, S. Microvessel density in follicular cysts, keratocystic odontogenic tumours and ameloblastomas. Asian Pac J Cancer Prev, v. 12, n. 2, p. 351-6, 2011.

30. SHEN, L. C. et al. Expression of osteonectin/secreted protein acidic and rich in cysteine and matrix metalloproteinases in ameloblastoma. J Oral Pathol Med, v. 39, n. 3, p. 242-9, 2010.

31. SIQUEIRA, A. S. et al. Matrix metalloproteinases, TIMPS and growth factors regulating ameloblastoma behaviour. Histopathology, v. 57, n. 1, p. 128-37, 2010.

32. SOUZA FREITAS, V. et al. Immunohistochemical expression of matrilysins (MMP-7 and MMP-26) in ameloblastomas and adenomatoid odontogenic tumors. Oral Surg Oral Med Oral Pathol Oral Radiol Endod, v. 108, n. 3, p. 417-24, 2009.

33. VERED, M. et al. Myofibroblasts in stroma of odontogenic cysts and tumors can contribute to variations in the biological behavior of lesions. Oral Oncol, v. 41, n. 10, p. 1028-33, 2005.

34. ZHANG, B. et al. Expression and role of metalloproteinase-2 and endogenous tissue regulator in ameloblastoma. J Oral Pathol Med, v. 39, n. 3, p. 219-22, 2010.

35. ZHANG, B. et al. Expression of RECK and matrix metalloproteinase-2 in ameloblastoma. BMC Cancer, v. 9, p. 427, 2009.

36. ZHANG, L. et al. Tissue inhibitor of metalloproteinase-2 inhibits ameloblastoma growth in a new mouse xenograft disease model. J Oral Pathol Med, v. 39, n. 1, p. 94-102, 2010.
Endereço para correspondência

Roseana de Almeida Freitas

Universidade Federal do Rio Grande do Norte Departamento de Odontologia

Av. Senador Salgado Filho, 1787 - Lagoa Nova

CEP: $59056-000$ - Natal-RN

Tel./fax: (84) 3215-4138 